\title{
A novel technique to prevent migration of esophageal stent
}

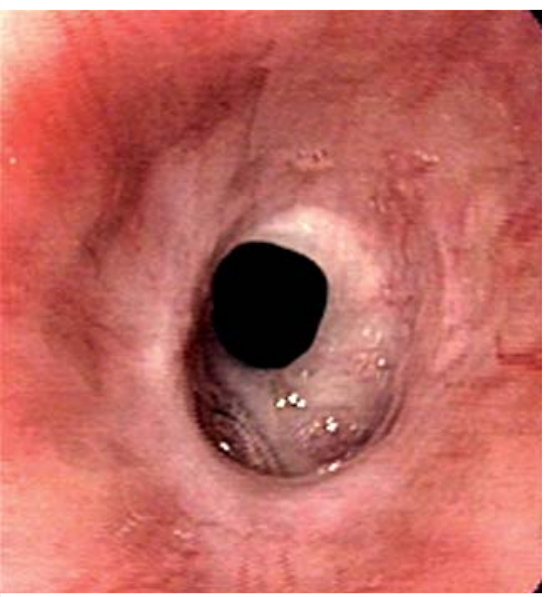

- Fig. 1 Nonulcerated esophageal stricture in the lower part of the esophagus.

A 25-year-old man presented with a history of dysphagia after corrosive ingestion. Upper gastrointestinal (GI) endoscopy revealed a stricture in the lower esophagus (\Fig.1), which persisted after repeated sessions of endoscopic dilatation. As a rescue treatment, a fully covered self-expandable metallic stent, $18 \mathrm{~mm}$ in diameter, was placed in the lower esophagus. To prevent slippage and migration of the stent, a novel technique was used to fix the stent to esophageal wall ( $\downarrow$ Video 1 ). The proximal end of the stent was fixed to the esophageal wall with clips. The procedure was performed under moderate sedation with Propofol. After submucosal injection with normal saline (stained with indigo carmine) at the proximal end of the stent, a vertical mucosal incision of $1 \mathrm{~cm}$ was made with a triangular tip knife ( $\triangleright$ Fig.2), and the mucosa distal to the incision was fixed to the stent by application of a hemoclip ( $>$ Fig. 3 ). Two clips were applied, one each at the 12 and 6 o'clock position, which fixed the stent to the esophageal wall ( $\triangleright$ Fig. 4 ). The whole procedure was well tolerated without any complication. After 4 weeks of follow-up, the stent was in situ and the patient asymptomatic.

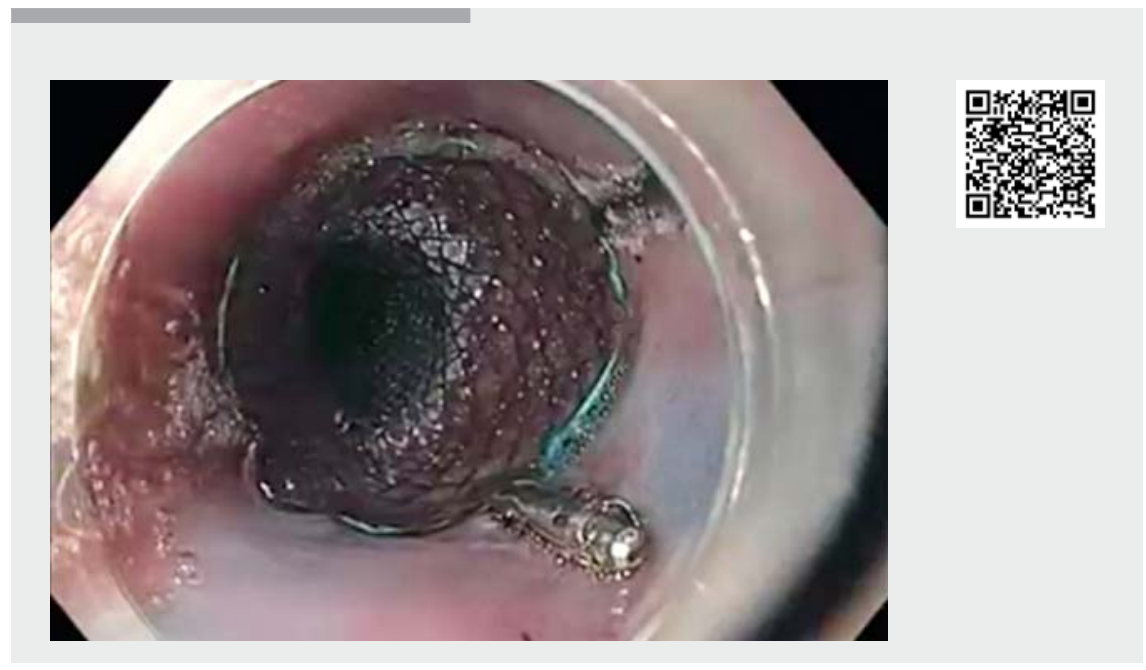

Video 1 Application of hemoclip to fix the self-expandable metal stent to the esophageal wall.

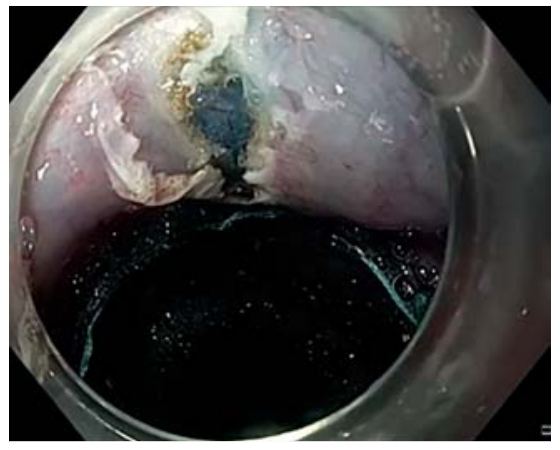

Fig. 2 Vertical mucosal incision at the 12 o'clock position.

Fully covered metal stents are being increasingly used for the benign esophageal diseases [1], however stent migration is not uncommon, and a migration rate of more than $30 \%$ has been reported [2]. Various methods such as suturing [3], external fixation with snare [4], and nasal fixation with silk thread [5] have been described. Hemoclips have been used previously to prevent migration [2] and reduced migration to $13 \%$. Fixing the clips after mucosal incision may better anchor the stent to the esophageal

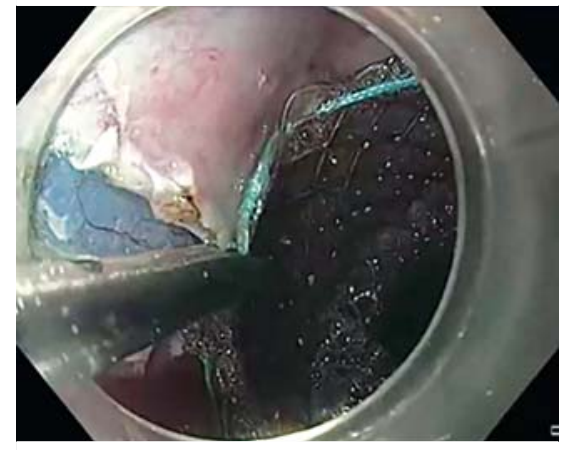

- Fig. 3 Application of hemoclip to fix the stent to the mucosa.

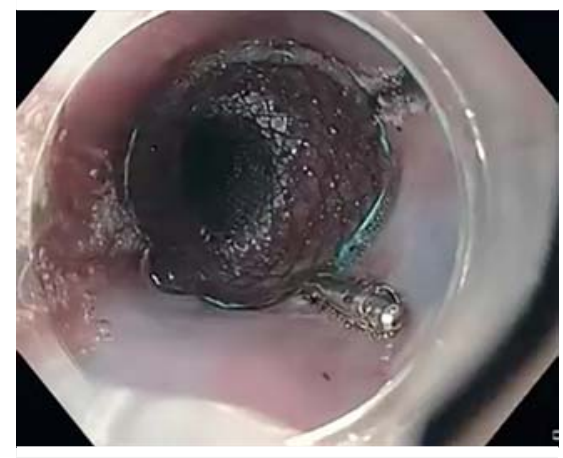

Fig. 4 Fixation of esophageal stent to the esophageal wall. 
wall and may further reduce the migration rate. Moreover, this technique can be performed with easily available equipment.

Endoscopy_UCTN_Code_TTT_1AO_2AZ

\section{Competing interests}

The authors declare that they have no con flict of interest.

The authors

Vikas Singla, Anil Arora, Shivam Khare, Ashish Kumar, Praveen Sharma, Naresh Bansal, Shrihari Anil Anikhindi

Institute of Liver, Gastroenterology and Pancreaticobiliary Sciences, Sir Ganga Ram Hospital, New Delhi, India
Corresponding author

\section{Vikas Singla, MD}

Institute of Liver, Gastroenterology and Pancreaticobiliary Sciences, Sir Ganga Ram Hospital, Rajinder Nagar, New Delhi, India 110060

singlavikas1979@gmail.com

\section{References}

[1] Thomas T, Abrams KR, Subramanian V et al. Esophageal stents for benign refractory strictures: a meta-analysis. Endoscopy 2011; 43: 386-393

[2] Vanbiervliet G, Filippi J, Karimdjee BS et al. The role of clips in preventing migration of fully covered metallic esophageal stents: a pilot comparative study. Surg Endosc 2012; 26: 53-59
[3] Kantsevoy SV, Bitner M. Esophageal stent fixation with endoscopic suturing device (with video). Gastrointest Endosc 2012; 76: 1251-1255

[4] Manes G, Corsi F, Pallotta S et al. Fixation of a covered self-expandable metal stent by means of a polypectomy snare: an easy method to prevent stent migration. Dig Liver Dis 2008; 40: 791-793

[5] Shim CS, Cho YD, Moon JH et al. Fixation of a modified covered esophageal stent: its clinical usefulness for preventing stent migration. Endoscopy 2001; 33: 843-848

Bibliography

Endoscopy 2020; 52: 1040-1041

DOI 10.1055/a-1149-1084

ISSN 0013-726X

Published online: 17.4.2020

(c) 2020. Thieme. All rights reserved. Georg Thieme Verlag KG Rüdigerstraße 14, 70469 Stuttgart, Germany 